\title{
Three phase induction motor modelling and control using vector control in LabVIEW
}

\author{
Wadee Khoury ${ }^{1, *}$, Aram Nasser ${ }^{1}$, and Péter Tamás Szemes ${ }^{2}$ \\ ${ }^{1}$ Masters Student, Debrecen University, Mechatronics Department, 4028 Debrecen, Hungary \\ ${ }^{2}$ Associate Professor, Debrecen University, Mechatronics Department, 4028 Debrecen, Hungary
}

\begin{abstract}
In this paper, a speed control system for a three-phase induction motor was modelled and designed within LabVIEW software environment. After structuring the dynamical model of the motor, a current controller was developed to stabilize the system and avoid a wind-up situation. Then, a speed controller was designed, using PID and Field Weakening techniques, to generate the reference current values. The field-weakening algorithm is used to achieve stability at speeds that are higher than the nominal one. An estimator is used to calculate the flux angle and the electromechanical speed of the motor. As a result, the system achieved the desired speed with good transient and steady state responses. In addition, the system proved to be robust when the torque load is applied in all cases.
\end{abstract}

\section{Introduction}

Three phase induction motors (IM), particularly squirrel cage, are rugged, efficient, and cheap compared to other motor types. Because of this, they are widely used in industry for many applications. In early days, due to the high cost and low efficiency of the IM speed control techniques, the use of the three-phase induction motor was limited to the applications that have constant speeds, such as driving fans and pumps. Following to the advancements in power electronics, IMs had become known as the industry horses and been more widely used in applications that require variable speed control $[1,2]$.

Simulating the control system behaviour before implementing it into a real world environment is convenient, and gives information about the control system efficiency and performance, as well as the system sensitivity against disturbances and noise signals.

In order to simulate the system and test it, the motor was modelled in LabVIEW using the T-model and the mathematical equations in the stationary reference frame, $\alpha \beta$. The vector control method, with PI and PID controllers, is used after converting the stationary variables $\alpha \beta$ into a rotating dq reference frame using the Park transform. The Park transformation needs the rotor flux angle as input. This angle is difficult to be measured, therefore, a current estimator was used to calculate it. Thanks to the field-weakening algorithm, described in [3], the system is able to operate on speeds higher than its nominal one and with good response. The system's simulation was tested on two different speeds (1400) [rpm], which is the nominal speed, and (2000) [rpm], with and without the torque load, where the system stability was evident.

\section{Induction Motor Modelling}

Several circuit representations are used to describe the motor's behaviour for transient and steady state analysis [4]. In this paper, the simple T-form of linear equivalent circuit in the stationary reference frame, shown in figure 1 , is used. This representation helps getting the state space that describes the IM's behaviour. By doing this, the complexity in the voltage equations of the IM can be simplified, resulted from the time varying mutual inductances between the stator and the rotor circuits [5]. To get the T-model, The IM's variables, currents, voltages, and fluxes, needed to be transferred into the stationary reference frame using Clarke Transform [6], given by:

$$
\left[\begin{array}{l}
i_{\alpha} \\
i_{\beta}
\end{array}\right]=\frac{2}{3}\left[\begin{array}{ccc}
1 & -\frac{1}{2} & -\frac{1}{2} \\
0 & \frac{\sqrt{3}}{2} & -\frac{\sqrt{3}}{2}
\end{array}\right]\left[\begin{array}{l}
i_{a} \\
i_{b} \\
i_{c}
\end{array}\right]
$$

Now, the dynamic model of the IM can be described in the stationary reference frame $\alpha \beta$, using the T-model shown in figure 1.

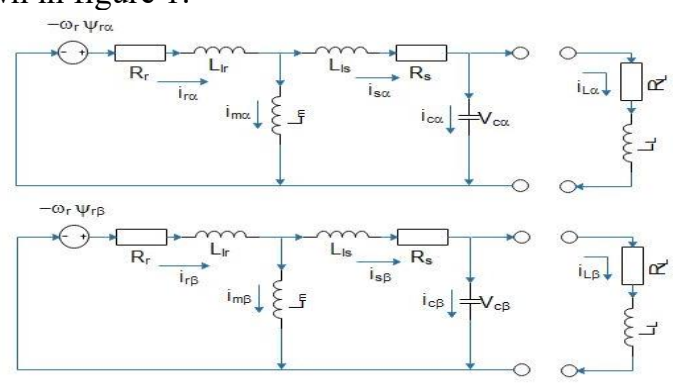

Fig. 1. The T-model equivelant circuite of the IM [1].

\footnotetext{
* Corresponding author: w.khoury@outlook.com
} 
From figure 1, the dynamical equations can be derived to get the IM's model [1].

$$
\begin{gathered}
v_{s \alpha}=R_{s} i_{s \alpha}+L_{s} \frac{d}{d t} i_{s \alpha}+L_{m} \frac{d}{d t} i_{r \alpha} \\
v_{s \beta}=R_{s} i_{s \beta}+L_{s} \frac{d}{d t} i_{s \beta}+L_{m} \frac{d}{d t} i_{r \beta} \\
0=R_{r} i_{r \alpha}+L_{r} \frac{d}{d t} i_{r \alpha}+L_{m} \frac{d}{d t} i_{s \alpha}+w_{r} \varphi_{r \beta} \\
0=R_{r} i_{r \beta}+L_{r} \frac{d}{d t} i_{r \beta}+L_{m} \frac{d}{d t} i_{s \beta}-w_{r} \varphi_{r \alpha}
\end{gathered}
$$

For a magnetically linear machine, the stator and rotor flux linkages are given by [4]:

$$
\left[\begin{array}{l}
\boldsymbol{\varphi}_{\boldsymbol{s}, \boldsymbol{\alpha} \boldsymbol{\beta}} \\
\boldsymbol{\varphi}_{\boldsymbol{r}, \boldsymbol{\alpha} \boldsymbol{\beta}}
\end{array}\right]=\left[\begin{array}{ll}
L_{s} & L_{m} \\
L_{m} & L_{r}
\end{array}\right]\left[\begin{array}{l}
\boldsymbol{i}_{\boldsymbol{s}, \boldsymbol{\alpha} \boldsymbol{\beta}} \\
\boldsymbol{i}_{\boldsymbol{r}, \boldsymbol{\alpha} \boldsymbol{\beta}}
\end{array}\right]
$$

Where:

- $\mathrm{v}_{\mathrm{s} \alpha}, \mathrm{v}_{\mathrm{s} \beta}$-Stator voltages in the stationary reference frame $[\mathrm{V}]$.

- $\mathrm{i}_{\mathrm{s} \alpha}, \mathrm{i}_{\mathrm{s} \beta}, \mathrm{i}_{\mathrm{r} \alpha}, \mathrm{i}_{\mathrm{r} \beta}-$ Stator and rotor currents in the stationary reference frame respectively $[\mathrm{A}]$.

- $\varphi_{\mathrm{r} \alpha}, \varphi_{\mathrm{r} \beta}-$ Rotor magnetic flux in the stationary reference frame $[\mathrm{Wb}]$.

- $\mathrm{R}_{\mathrm{s}}, \mathrm{R}_{\mathrm{r}}-$ Resistances of the IM's stator and rotor respectively $[\Omega]$.

- $\mathrm{L}_{\mathrm{s}}, \mathrm{L}_{\mathrm{r}}-$ Self-inductances of the IM's stator and rotor respectively $[\mathrm{H}]$.

- $\mathrm{L}_{\mathrm{m}}$-Mutual inductance between the stator and the rotor $[\mathrm{H}]$.

- $\quad w_{\mathrm{r}}$-Rotor speed [rad/s].

As the self-inductance of the stator and rotor are composed of the leakage and mutual inductances as [7]:

$$
L_{s}=L_{r}=L_{l s}+L_{m}
$$

The mechanical behaviour can be expressed as:

$$
\begin{aligned}
\frac{J}{n_{p}} \frac{d w_{r}}{d t} & =T_{e}-T_{L} \\
\frac{d \theta}{d t} & =w_{r}
\end{aligned}
$$

And the electrical torque developed by the IM:

$$
T_{e}=\frac{3 n_{p}}{2} L_{m}\left(i_{r \alpha} i_{s \beta}-i_{r \beta} i_{s \alpha}\right)
$$

When considering the coefficient of friction, $B \neq 0$, the load is expressed as:

$$
T_{L}=B \frac{w_{r}}{n_{p}}+T_{L, \text { extra }}
$$

Where:

$$
\left.\mathrm{J} \text { : Moment of inertia [Kg. } \mathrm{m}^{2}\right]
$$

$\theta$ : Angular position [rad]

$n_{p}$ : Number of pole pairs

By combining equations (2-10), the comprehensive equation of the IM [7] is obtained and can be represented in the following form:

$$
u=R x+L \dot{x}
$$

$$
\left[\begin{array}{c}
V_{s \alpha} \\
V_{s \beta} \\
0 \\
0
\end{array}\right]=[\mathrm{R}]\left[\begin{array}{c}
i_{s \alpha} \\
i_{s \beta} \\
i_{r \alpha} \\
i_{r \beta}
\end{array}\right]+[\mathrm{L}]\left[\begin{array}{c}
\frac{d i_{s \alpha}}{d t} \\
\frac{d i_{s \beta}}{d t} \\
\frac{d i_{r \alpha}}{d t} \\
\frac{d i_{r \beta}}{d t}
\end{array}\right]
$$

Where:

$$
\begin{gathered}
{[R]=\left[\begin{array}{cccc}
R_{s} & 0 & 0 & 0 \\
0 & R_{s} & 0 & 0 \\
0 & w_{r} L_{m} & R_{r} & w_{r} L_{r} \\
-w_{r} L_{m} & 0 & -w_{r} L_{r} & R_{r}
\end{array}\right]} \\
{[L]=\left[\begin{array}{cccc}
L_{s} & 0 & L_{m} & 0 \\
0 & L_{s} & 0 & L_{m} \\
L_{m} & 0 & L_{r} & 0 \\
0 & L_{m} & 0 & L_{r}
\end{array}\right]}
\end{gathered}
$$

In order to get the IM state-space model, equation (13) is rearranged to be in the form [7]:

$$
\dot{x}=-L^{-1} R x+L^{-1} u=A x+B u
$$

\section{Control Method}

\subsection{VECTOR CONTROL}

Two major control methods are used to control the IM's speed: the scalar control, referred to as $\mathrm{V} / \mathrm{Hz}$, and the vector control, also known as field-oriented control, referred to as FOC. The first method is cheap, easy to implement, and gives good steady state results. However, this method gives slow transient response; therefore, it is not efficient in controlling dynamical systems [5]. On the other hand, although it is more complex, FOC has fast and excellent transient response [8]. Because of this, vector control method has become more popular in industry for the speed and torque control of the IM.

The idea behind the vector control method is to identify the stator currents of an IM as two orthogonal components that can be visualized with a vector, which gives the ability to deal with the IM as a separately excited two-phase DC machine. One component $\left(\mathrm{i}_{\mathrm{q}}\right)$ refers to a virtual current that controls the torque and the other component $\left(i_{d}\right)$ refers to a virtual current that controls the flux [9]. As they are decoupled or orthogonal in space, the torque and the flux can be controlled separately without affecting one another.

To apply the FOC, the rotor flux angle is needed, and since this angle is hard to be measured, a flux estimator is used to calculate it. 


\subsection{Flux Estimator}

There are two basic ways to determine the angle of the rotor flux. The direct scheme, which needs field sensors to measure the rotor flux position directly. This scheme is difficult, or maybe impossible to implement [5]. Instead, the indirect scheme can be used, which uses equations to estimate the rotor flux angle mathematically from other measurable components. For the indirect estimation, either the current based flux or voltage based flux can be used [7]. In this paper, the measured current is taken to estimate the rotor flux angle, as well as the synchronous speed of the IM.

By introducing a new constant, Leakage coefficient $(\sigma)$, as in equation (15), and rearranging equations (2-5), the stator and rotor flux equations can be written as [10]:

$$
\begin{gathered}
\sigma=1-L_{s} i_{s \alpha} \\
\varphi_{s \alpha}=\int\left(v_{s \alpha}-R_{s} i_{s \alpha}\right) d t \\
\varphi_{s \beta}=\int\left(v_{s \beta}-R_{s} i_{s \beta}\right) d t \\
\varphi_{r \alpha}=\frac{L_{r}}{L_{m}}\left(\varphi_{s \alpha}-\sigma L_{s} i_{s \alpha}\right) \\
\varphi_{r \beta}=\frac{L_{r}}{L_{m}}\left(\varphi_{s \beta}-\sigma L_{s} i_{s \beta}\right)
\end{gathered}
$$

The rotor and stator flux angles can now be calculated as

$$
\begin{aligned}
& \theta_{\varphi s}=\tan ^{-1} \frac{\varphi_{s \beta}}{\varphi_{s \alpha}} \\
& \theta_{\varphi r}=\tan ^{-1} \frac{\varphi_{r \beta}}{\varphi_{r \alpha}}
\end{aligned}
$$

From equation (20), the synchronous speed is:

$$
w_{1}=\theta_{\varphi s}^{\cdot}=\frac{\varphi_{s \alpha} \varphi_{s \beta}^{\cdot}-\varphi_{s \beta} \varphi_{s \alpha}^{\cdot}}{\varphi_{s \alpha}^{2}+\varphi_{s \beta}^{2}}
$$

\subsection{Park Transformation}

The estimated rotor flux angle is used to transform the stationary reference frame into the rotating reference frame using the Park transform [6]:

$$
\left[\begin{array}{l}
i_{d} \\
i_{q}
\end{array}\right]=\left[\begin{array}{cc}
\cos \theta & \sin \theta \\
-\sin \theta & \cos \theta
\end{array}\right]\left[\begin{array}{l}
i_{\alpha} \\
i_{\beta}
\end{array}\right]
$$

Or, in case of designing using complex numbers, the following formula can be considered:

$$
v_{d q}=v_{\alpha \beta} e^{-j \theta}
$$

Where $j=\sqrt{-1}$ is the complex number.

\subsection{Speed control model}

The $\mathrm{i}_{\mathrm{q}}$ component is controlled using a parallel PID controller after getting the error signal, which is the difference between the measured mechanical speed and the desired one. To satisfy the design requirements, the controller parameters were found as follow:

TABLE I. PID PARAMETERS

\begin{tabular}{|c|c|}
\hline Controller Parameters & Value \\
\hline $\boldsymbol{k}_{\boldsymbol{p}}$ & 30 \\
\hline $\boldsymbol{k}_{\boldsymbol{i}}$ & 0.35 \\
\hline $\boldsymbol{k}_{\boldsymbol{d}}$ & 0.04 \\
\hline
\end{tabular}

\subsubsection{Generation of the $d$ current component}

To generate the desired $i_{d}$ component, the field weakening technique is used. This technique allows to operate the IM on speeds higher than its nominal one by reducing the flux in the air-gap between the stator and rotor, which in turn reduces the back emf constant, and hence the torque. From the field weakening equations (24) and (25), the reference rotor flux is generated using the inverse- $\Gamma$-model, described in [3], as follow:

$$
\begin{gathered}
\varphi_{r, \text { ref }}=\int_{\varphi_{r, \text { min }}}^{\varphi_{r, \text { rated }}} k\left(v_{\text {base }}{ }^{2}-v_{d, r e f}{ }^{2}-v_{q, r e f}{ }^{2}\right) d t \\
k=\frac{\propto_{f} L_{m}}{2 w_{f} L_{\sigma} v_{s, \text { rated }}} \\
L_{\sigma}=L_{s}-\frac{L_{m}}{L_{r}}
\end{gathered}
$$

Where:

- $\mathrm{V}_{\text {base }}$-Maximum peak voltage that the power electronics can put out (326.6 [v]).

- $v_{\mathrm{d}, \mathrm{ref}}, \mathrm{v}_{\mathrm{q}, \mathrm{ref}}-$ Reference voltage values from the voltage controller.

- $\quad \propto_{f}-$ A constant bandwidth of the field-weakening algorithm [0.5].

- $L_{\sigma}$-Leakage inductance variable from the inverse- $\Gamma$-model.

- $w_{f}$ is given by:

$$
w_{f}=\left\{\begin{array}{cll}
w_{1, \text { rated }} & \text { if } & \left|w_{1}\right| \leq w_{1, \text { rated }} \\
\left|w_{1}\right| & \text { if } & \left|w_{1}\right|>w_{1, \text { rated }}
\end{array}\right.
$$

The output of the field-weakening algorithm, which is the reference $i_{d}$ component, is calculated using the following equation:

$$
i_{d, r e f}=\frac{\varphi_{r, r e f}}{L_{m}}
$$

The Field-Weakening method is illustrated in figure 2.

\subsubsection{Generation of the q current component}




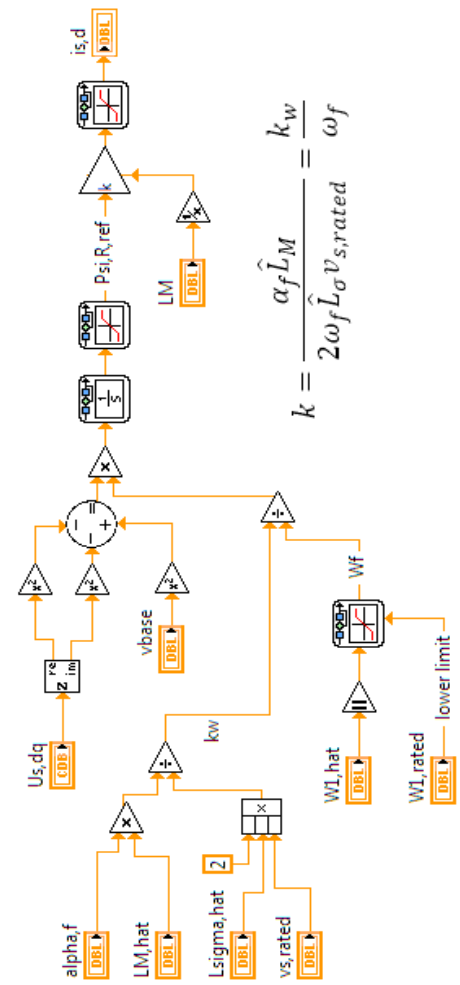

Fig. 2. Field wakening block diagram.

\subsubsection{Current Controller model}

The resulting currents $i_{\text {s.dq }}$ from the Park transformation will be compared with the reference $\mathrm{i}_{\mathrm{s.dq}, \mathrm{ref}}$; the output of the speed controller, and the error signal will be fed to the current controller. Using a normal PI controller might cause the system to wind-up when the voltage is saturated by its maximum value. If the reference voltage gets higher than the voltage limit, there will be an error between the limited voltage and the actual voltage. This causes the current to increase slower, which makes the integrator integrates too much. When the current reaches the reference value, the accumulated error will be too large, causing an overshoot. To negate this, a back calculation algorithm is used by introducing a new error, $\overline{\mathrm{e}}$, which will be fed to the integrator, using the following equations [7]:

$$
\bar{e}=e+\frac{\bar{v}_{s, l i m}-v_{S}}{k_{p c}}
$$

Where:

$$
\begin{gathered}
\bar{v}_{s, \text { lim }}=\left\{\begin{array}{ccc}
v_{s} & \text { if } & \left|v_{s}\right| \leq v_{s, \max } \\
\left|v_{s, \max }\right|<v_{s} & \text { if } & \left|v_{s}\right|>v_{s, \max }
\end{array}\right. \\
v_{s, \text { dq,out }}=e k_{p c}+\int \bar{e} k_{i c}
\end{gathered}
$$

$\mathrm{k}_{\mathrm{pc}}, \mathrm{k}_{\mathrm{ic}}-\mathrm{PI}$ controller gains, and their values are 27 and 6400 respectively.

Finally, the output voltage is transferred to the threephase equivalent reference frame using the inverse Clarke-Park transformations to feed the motor with the proper signals.

\section{LabVIEW Simulation}

LabVIEW simulation loop was used for modelling and controlling the three phase induction motor, where the dynamical model, represented in state-space form, is implemented as shown in figure 3 .

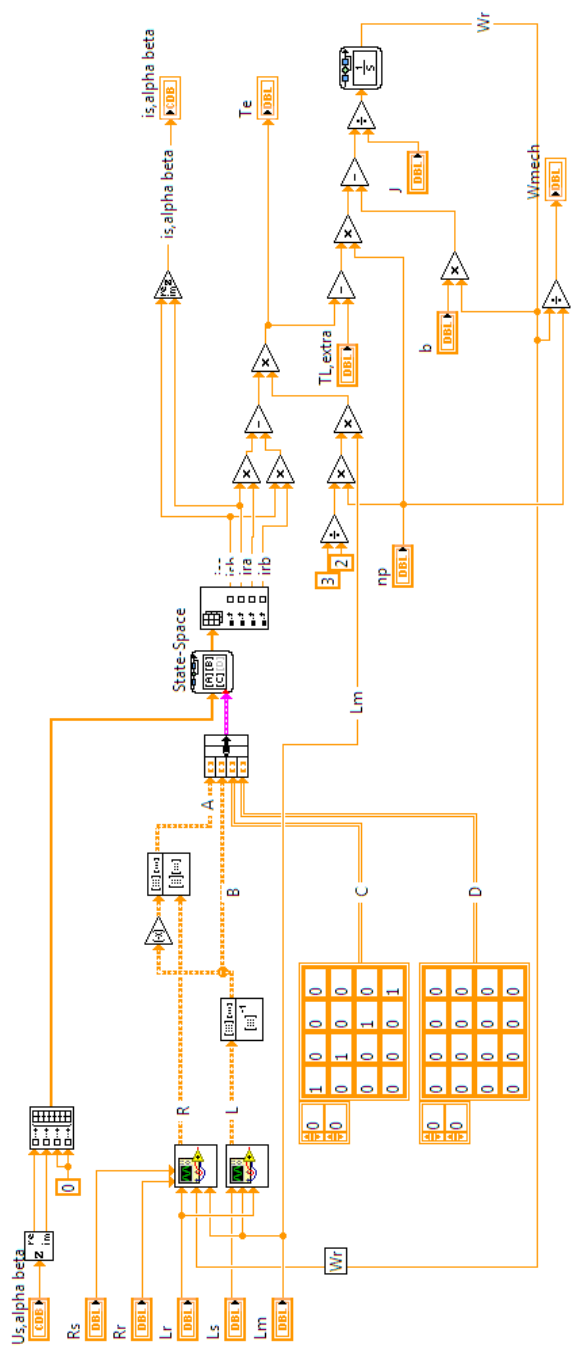

Fig. 3. AC Induction Motor block diagram.

The IM model parameters used in the simulation are described in the following table.

TABLE II. MOTOR PARAMETERS

\begin{tabular}{|c|c|}
\hline Parameter [unit] & Value \\
\hline Stator inductance $\boldsymbol{L}_{\boldsymbol{s}}[\boldsymbol{H}]$ & 0.1276 \\
\hline Rotor inductance $\boldsymbol{L}_{\boldsymbol{r}}[\boldsymbol{H}]$ & 0.1276 \\
\hline Mutual inductance $\boldsymbol{L}_{\boldsymbol{m}}[\boldsymbol{H}]$ & 0.1186 \\
\hline Stator resistance $\boldsymbol{R}_{\boldsymbol{s}}[\Omega]$ & 2.4 \\
\hline Rotor resistance $\boldsymbol{R}_{\boldsymbol{r}}[\Omega]$ & 2.3 \\
\hline Number of pole pairs $\boldsymbol{n}_{\boldsymbol{p}}$ & 2 \\
\hline Coefficient of friction $\boldsymbol{B}$ & 0.003 \\
\hline Moment of inertia $\boldsymbol{J}\left[\boldsymbol{K g} \cdot \boldsymbol{m}^{\mathbf{2}}\right]$ & 0.00529 \\
\hline
\end{tabular}


The complete LabVIEW implementation of the control system is presented in figure 4 .

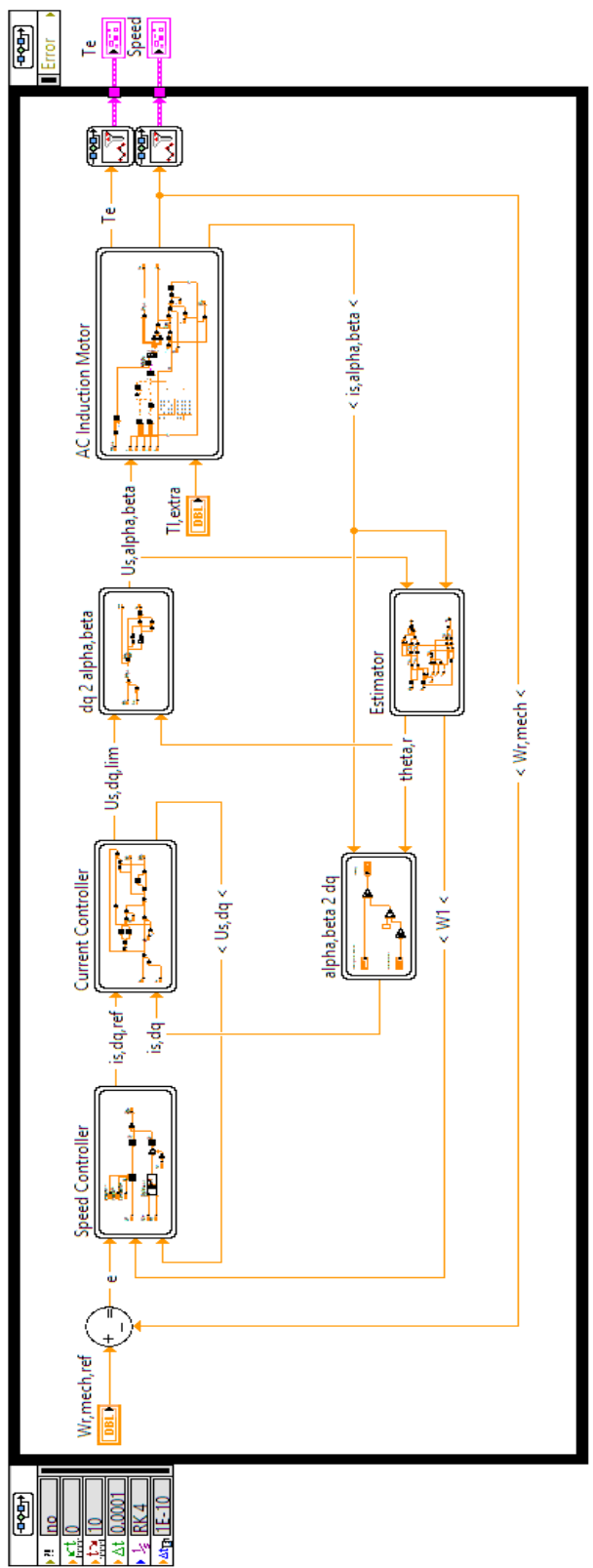

Fig. 4. Full control system block diagram.

First, the system is simulated with a desired speed of 1400 [rpm], which is the rated speed of the motor. The speed response is shown in figure 5 .

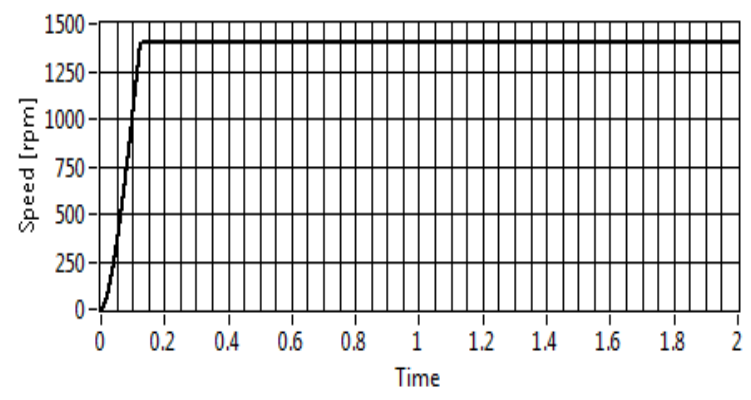

Fig. 5. Mechanical speed at $w_{\text {mech,ref }}=1400[\mathrm{rpm}]$.
As a result of this simulation, the speed reaches its final value without any overshoot and only within 0.1 second, which means that the control system is fast and has a good transient response. As the load torque is applied at the first second, the system behaviour remains the same without any change in the speed value, allowing the motor to work at a constant speed with or without the load, proving the robustness of the system.

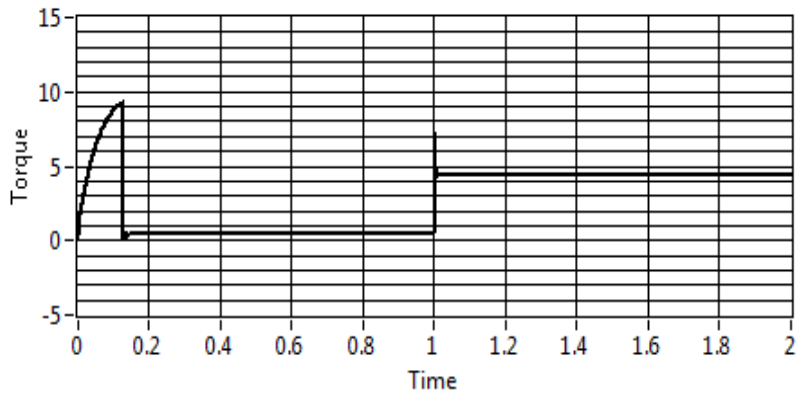

Fig. 6. Electromechanical torque at $w_{\text {mech, } r e f}=1400[\mathrm{rpm}]$.

To keep the motor's speed constant, current components change while working on the transient state as shown in figure 7 , to become stable at steady state. Then, they change again, especially $i_{q}$, when applying the torque at the first second. These components affect the motor's torque and flux, as shown in figure 6, and therefore, maintaining a constant motor speed.

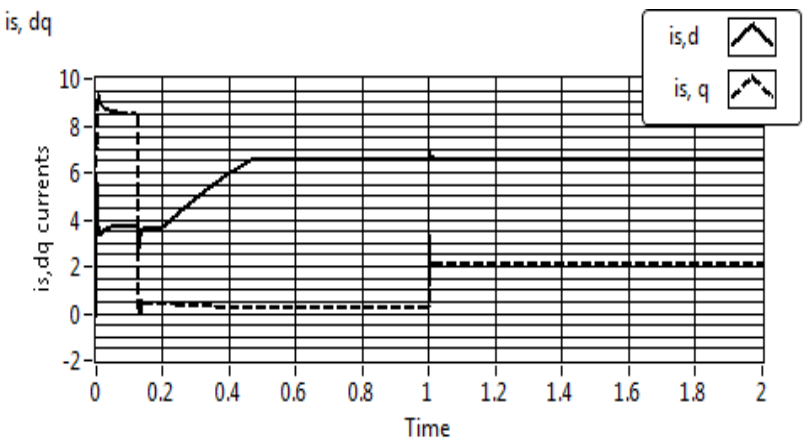

Fig. 7. Current components at $w_{\text {mech, ref }}=1400[\mathrm{rpm}]$.

In order to test the effectiveness of the field weakening technique, the system is simulated with a desired mechanical speed of 2000 [rpm], higher than the rated speed, 1400 [rpm]. The field weakening will reduce the $\mathrm{d}$ current component, which will decrease the flux in the airgap, causing higher speeds. The speed response is presented in figure 8 .

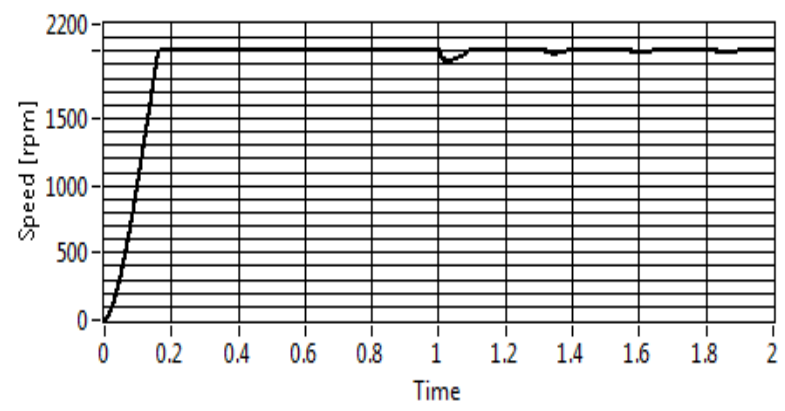

Fig. 8. Mechanical speed at $w_{\text {mech,ref }}=2000[\mathrm{rpm}]$. 


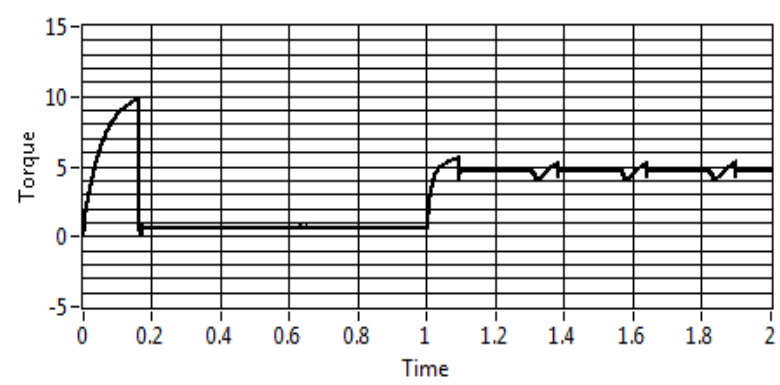

Fig. 9. Electromechanical torque at $w_{\text {mech,ref }}=2000[\mathrm{rpm}]$.

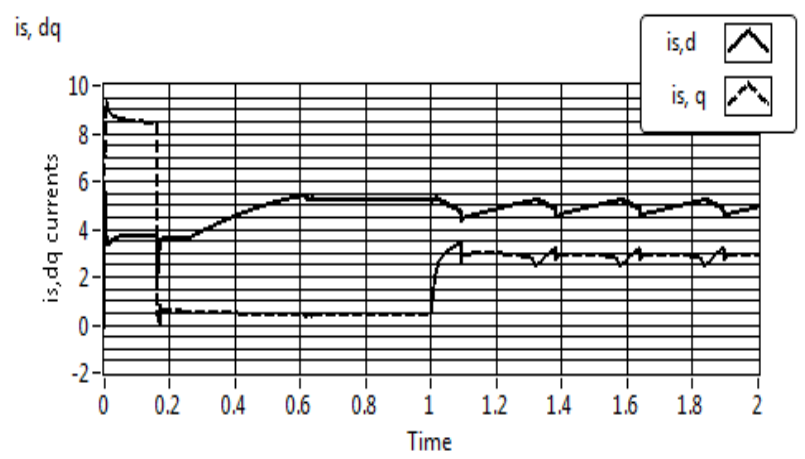

Fig. 10. Current components at $w_{\text {mech, ref }}=2000[\mathrm{rpm}]$.

As a result, in figure 8 , the speed reaches its desired value within $0.4 \mathrm{sec}$ that is larger than the one in the previous simulation. This response delay is due to the fact that the field weakening block is reducing $i_{d}$, as shown in figure 10, comparing with the nominal value in figure 7 . This in turn will affect in reducing the flux, back EMF constant, and torque, resulting in achieving the high speed.

Additionally, the existence of small fluctuations in figure 8 is due to applying a high load, 7 [N.m], when the motor was working in the field weakening region. Therefore, the field weakening technique should be used in applications where the applied load is expected to be small. Nevertheless, although a high torque was applied in the field weakening region, the control system tried to keep the desired speed constant, which reflects the robustness of the system. With real motors, however, this might not be totally true, where the motor's heating problems should be taken into consideration while designing and implementing the control system.

\section{Conclusion}

As discussed earlier, LabVIEW software is used to model and simulate the speed control of a three-phase induction motor with and without the extra load. At normal speeds, the system achieved good response and proved to be robust when the load was applied. At speeds higher than the nominal one, the field weakening technique was able to achieve good response with being carful not to operate the system in applications that require high toque. The simulation results show the efficiency of using the vector control and field-weakening methods.

\section{Acknowledgment}

The work/publication is supported by the EFOP-3.6.116-2016-00022 project. The project is co-financed by the European Union and the European Social Fund.

\section{References}

1. K. Sundararaju, R.S. Kumar, and I.G.C. Raj, Modeling and simulation of neural based speed controller for direct torque control of three phase induction motor. In Region 10 Conference, TENCON, 1439-1444 (2017). IEEE.

2. S. Hegde, S. Angadi, A.B. Raju, Speed control of 3phase induction motor using volt/hertz control for automotive application, I4C, 17214371, (2017). IEEE.

3. L. Harnefors, K. Pietilainen, L. Gertmar, Torquemaximizing field-weakening control: design, analysis, and parameter selection, IEEE, Transactions on Industrial Electronics, 48(1), 161168, (2001).

4. G.R. Slemon, Modelling of induction machines for electric drives, IEEE Transactions on Industry Applications, 25(6), 1126-1131, (1989).

5. M.R.B. Fathima, P.M.J. Princy, S. RamPrasath, Mathematical modeling of SVPWM inverter fed 3 phase induction motor vector control in MATLAB/Simulink environment, In Circuit, Power and Computing Technologies (ICCPCT), 2017 International Conference on, 1-8, (2017). IEEE.

6. J.M. Peña, E.V. Díaz, Implementation of V/f scalar control for speed regulation of a three-phase induction motor, In ANDESCON, 1-4, (2016). IEEE.

7. J. Bj “orkqvist, D. Stockman, Implementation and simulation of sensorless control and field weakening for an induction machine Using the the Statically Compensated Voltage Model, Department of Energy and Environment Division of Electric Power Engineering, Chalmers University of Technology, Master's thesis, (2014).

8. G. Kohlrusz, D. Fodor, Comparison of scalar and vector control strategies of induction motors, Hungarian Journal of Industry and Chemistry, 39(2), 265-270 (2011)

9. M.S. Aspalli, S.J. Patil, Study of AI and PI controller using SVPWM technique for induction motor speed control, In Electrical, Electronics, Communication, Computer, and Optimization Techniques (ICEECCOT), 2017 International Conference on, 16, (2017). IEEE.

10. P. Parida, A Sliding Mode Controller For Induction Motor Drives. National Institute of Technology in Rourkela, Doctoral dissertation (2009). 